\title{
Nonlinear force-free reconstruction of the global solar magnetic field: methodology
}

\author{
I. Contopoulos • C. Kalapotharakos . \\ M. K. Georgoulis
}

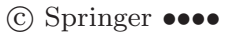

\begin{abstract}
We present a novel numerical method that allows the calculation of nonlinear force-free magnetostatic solutions above a boundary surface on which only the distribution of the normal magnetic field component is given. The method relies on the theory of force-free electrodynamics and applies directly to the reconstruction of the solar coronal magnetic field for a given distribution of the photospheric radial field component. The method works as follows: we start with any initial magnetostatic global field configuration (e.g. zero, dipole), and along the boundary surface we create an evolving distribution of tangential (horizontal) electric fields that, via Faraday's equation, give rise to a respective normal field distribution approaching asymptotically the target distribution. At the same time, these electric fields are used as boundary condition to numerically evolve the resulting electromagnetic field above the boundary surface, modelled as a thin ideal plasma with non-reflecting, perfectly absorbing outer boundaries. The simulation relaxes to a nonlinear force-free configuration that satisfies the given normal field distribution on the boundary. This is different from existing methods relying on a fixed boundary condition - the boundary evolves toward the a priori given one, at the same time evolving the three-dimensional field solution above it. Moreover, this is the first time a nonlinear force-free solution is reached by using only the normal field component on the boundary. This solution is not unique, but depends on the initial magnetic field configuration and on the evolutionary course along the boundary surface. To our knowledge, this is the first time that the formalism of force-free electrodynamics, used very successfully in other astrophysical contexts, is applied to the global solar magnetic field.
\end{abstract}

Keywords: Magnetic fields, Corona; Active Regions, Magnetic Fields

Research Center for Astronomy and Applied Mathematics (RCAAM), Academy of Athens, 4 Soranou Efessiou Str.,

Athens 11527, Greece, email: icontop@academyofathens.gr 


\section{Introduction}

Force-Free Electrodynamics (hereafter FFE) is a formal name for time-dependent electromagnetism in an ideal plasma with negligible inertia $(\rho=0)$ and negligible gas pressure $(\beta=0)$. The formalism of FFE has been developed for various relativistic astrophysical applications (pulsars, astrophysical jets, gamma-ray bursts, etc.) where the plasma supports electric currents and electric charges (Gruzinov, 1999). The equations of FFE are Maxwell's equations with nonzero electric fields as follows:

$$
\begin{gathered}
\frac{\partial \mathbf{E}}{\partial t}=c \nabla \times \mathbf{B}-4 \pi \mathbf{J}, \\
\frac{\partial \mathbf{B}}{\partial t}=-c \nabla \times \mathbf{E} \\
\nabla \cdot \mathbf{B}=0
\end{gathered}
$$

These equations are coupled by the ideal MHD condition

$$
\mathbf{E} \cdot \mathbf{B}=0
$$

implying that in an ideal plasma $\mathbf{E}$ and $\mathbf{B}$ are everywhere perpendicular, and the force-free condition

$$
\rho_{e} \mathbf{E}+\frac{1}{c} \mathbf{J} \times \mathbf{B}=0
$$

Here, $\mathbf{J}$ is the electric current density, and $\rho_{e} \equiv(4 \pi)^{-1} \nabla \cdot \mathbf{E}$ is the electric charge density. Gruzinov (1999) showed that it is possible to solve for $\mathbf{J}$ in the above set of equations and thus express the electric current density as a function of the electric and magnetic field, namely

$$
\mathbf{J}=\frac{c}{4 \pi} \nabla \cdot \mathbf{E} \frac{\mathbf{E} \times \mathbf{B}}{B^{2}}+\frac{c}{4 \pi} \frac{(\mathbf{B} \cdot \nabla \times \mathbf{B}-\mathbf{E} \cdot \nabla \times \mathbf{E})}{B^{2}} \mathbf{B} .
$$

One can then numerically integrate Maxwell's equations (eqs 1, 2) to obtain the time evolution of the electric and magnetic fields. We developed a threedimensional (hereafter 3D) finite-difference time-domain (FDTD) cartesian FFE code with non-reflecting, perfectly absorbing outer boundaries applied successfully to the 3D structure of the pulsar magnetosphere (Kalapotharakos and Contopoulos, 2009, see also Yee, 1966; Spitkovsky, 2006).

We then realized that the same formalism may be applied to follow the temporal evolution of any open force-free ideal-MHD system from an initial to a final magnetostatic configuration (i.e. with zero initial and final electric fields) when the magnetic field distribution at a boundary evolves for a finite number of time-iteration steps. After sufficient time, when the magnetic field at the boundary will have reached a given distribution, and all electric fields will 
have relaxed to zero, the above expressions degenerate into the following limited set of steady-state FFE equations,

$$
\begin{gathered}
\mathbf{J}=\frac{c}{4 \pi} \nabla \times \mathbf{B}, \\
\nabla \cdot \mathbf{B}=0, \text { and } \\
\mathbf{J} \times \mathbf{B}=0 .
\end{gathered}
$$

A solution of the system of eqs. (7)-(9) satisfying a given normal-field distribution on the boundary can be viewed as the final FFE equilibrium solution. Given the ill-posed nature of this problem (only the normal field component on the boundary is known), this solution is not unique and depends on the initial magnetostatic configuration and on the course toward equilibrium 1 .

The above methodology can be applied to the inner solar corona, that is, between 1 and $2-2.5 r_{\odot}$ (solar radii). In this environment, motions in the linetying photosphere largely but nonlinearly dictate the evolution in the magnetic field lines above, along which plasma is assumed 'frozen in' and forced to follow their path $(\beta \ll 1)$. At the edge of the inner corona, known as the 'source surface' (Schatten, Wilcox, and Ness, 1969) the plasma again approaches equipartition with the magnetic field. Beyond the source surface the plasma starts dominating again in the form of the solar wind. Therefore, between $1 r_{\odot}$ (photosphere) and $\sim 2.5 r_{\odot}$ (source surface) the magnetic field is thought to approximately satisfy eqs. (7)-(9) along with the divergence-free condition, eq. (3), and the ideal MHD condition, eq. (47). In discussing the global solar field, and driven by the necessity to use the observed photospheric magnetic field as the boundary condition, we ignore the fact that the latter is, in fact, forced, rather than force-free (Metcalf et al., 1995; Georgoulis and LaBonte, 2004).

Routine measurements of the global photospheric field provide only the lineof-sight magnetic field component $B_{L O S}\left(r_{\odot}, \theta, \phi\right)$ for given heliocentric spherical coordinates $(\theta, \phi) 2$. This is typically accomplished by synoptic (Carrington) maps of $B_{L O S}$ where the heliographic latitude and the Carrington longitude can be transformed into $\theta$ and $\phi$, respectively. Synoptic maps take one solar rotation ( $\sim 27$ days at the equator) to complete one Carrington rotation $\left(0^{\circ}\right.$ - $360^{\circ}$ in Carrington longitude) but are again assumed to capture a snapshot of the photospheric magnetic field for practical reasons (see, however, the attempt to evolve the photospheric field by means of a flux dispersal model (Schrijver and De Rosa, 2003) and use the modified boundary for global field extrapolations).

\footnotetext{
${ }^{1}$ The above statements are correct mathematically. In practice, however, as shown in $\S 3$, numerical dissipation sets in and the system evolves adiabatically through a sequence of nonlinear force-free magnetostatic equilibria with decreasing magnetic free energy towards a unique, minimum-energy and hence current-free (potential) equilibrium.

${ }^{2}$ The Vector SpectroMagnetograph (VSM; Henney et al. 2009) of the Synoptic Optical Long Term Investigations of the Sun (SOLIS) facility and the Helioseismic and Magnetic Imager (HMI) onboard the Solar Dynamics Observatory (SDO) is and will be providing, respectively, the first full-disk photospheric vector magnetograms.
} 
Determining the distribution of coronal magnetic fields and subsequent electric currents is, per the above, a formidable task. Global solar field extrapolations are current-free (potential) in their majority (Altschuler and Newkirk, 1969 Wang and Sheeley, 1992: Luhmann et al., 2002, Schrijver and De Rosa, 2003). Some magnetohydrodynamic modelling efforts are also present in the literature (Linker et al., 1999, Riley, Linker, and Mikić, 2001. Roussev et al., 2003) but they require immense computing resources. To our knowledge, the only technique providing a global solar nonlinear force-free field is the optimization method of T. Wiegelmann and collaborators. A magnetostatic version of this method was proposed by Wiegelmann (2007). The main idea was to simultaneously minimize the Lorentz force $(\nabla \times \mathbf{B}) \times \mathbf{B}$ and the divergence $(\nabla$. $\mathbf{B})$ of the field in the extrapolation volume in spherical coordinates - a generalization of the Cartesian implementation of Wiegelmann (2004). Efforts to further refine the method are discussed in Tadesse, Wiegelmann, and Inhester (2009) while a further generalization into a magnetohydrostatic nonlinear force-free solution is described by Wiegelmann et al. (2007) and Ruan et al. (2008). These optimization efforts, however, generally require the vector magnetic field on the photospheric boundary. We hereby propose an alternative approach reaching a nonlinear force-free magnetostatic solution by only using the normal (radial) field component on the boundary, as follows:

i) Start with a simple static potential magnetic field distribution such as $\mathbf{B}=0$ everywhere, or a dipolar field of any direction, etc.

ii) Evolve the vertical photospheric magnetic field by imposing a distribution of horizontal photospheric electric fields using Faraday's equation (eq. 2). This distribution is chosen such that the resulting photospheric magnetic field asymptotically $(t \rightarrow \infty)$ approaches a given photospheric magnetogram (see next section).

iii) During the evolution of the photospheric magnetic field, force-free electrodynamic waves are injected from the photosphere into the corona. Assuming non-reflecting, perfectly absorbing conditions at large distances, these waves will be absorbed by the outer boundaries and will not re-enter the computational domain. The moving electric charges carried by these waves begin to establish a nonlinear network of coronal electric currents.

iv) Gradually, as the photospheric magnetic field distribution approaches the target, photospheric electric fields will correspondingly decrease, and as a consequence magnetospheric electrodynamic waves, electric fields, and electric charges will also asymptotically fade. The nonlinear network of coronal electric currents, however, will survive.

$v$ ) When electric fields vanish everywhere, we relax to a force-free configuration that closely matches the given photospheric boundary condition.

At this initial stage we cannot claim that our solution is representative of the actual global coronal field. In fact, and as argued above, our solution depends on the initial condition and on the course followed to reproduce the given boundary condition (see, however, footnote 1). To achieve a physically meaningful equilibrium solution we need to apply additional physical arguments that will be the subject of a future publication. This work simply lays out the details of the methodology and presents a few characteristic test cases. Notice that our 
methodology does not apply in studying the dynamical evolution of the solar corona between successive photospheric configurations (the characteristic speed of propagation of information in the solar corona, the Alfvèn speed, is on the order of $1,000 \mathrm{~km} / \mathrm{sec}$, whereas the characteristic speed of propagation of information in our electrodynamic 'corona', the speed of light, is $\sim 300$ times faster). It does apply, though, in determining the equilibrium coronal configuration.

Our method has certain similarities with 'stress-and-relax' schemes proposed by e.g. Roumeliotis (1996), Valori, Kliem, and Keppens (2005), in that we both act on the boundary magnetic field distribution, and then evolve the magnetospheric field towards stationarity. Our respective technical implementations, though, are different (e.g. they work with the vector potential, we work only with the magnetic field; they 'stress' the horizontal boundary magnetic field component, we evolve the radial one; they rely on viscous damping in order to settle to stationarity, we implement absorbing outer boundary conditions that remove any non-stationarity from our computational box).

In $\S 2$ we present our numerical implementation of a photospheric electric field distribution which guarantees that the distribution of the radial photospheric magnetic field component will asymptotically approach that of a given solar magnetogram. In $\S 3$ we follow the electrodynamic response of the corona under the action of the above photospheric electric field distribution, and present some representative solutions for various initial conditions. We also present the various quantities used to monitor the course toward equilibrium. Our conclusions are summarized and discussed in $\S 4$.

\section{The photospheric magnetic field}

Let us first assume a local 2D staggered cartesian grid $(x, y)$ of size element $\delta$ in the photosphere, with normal magnetic field $B_{z}$ defined at the center of each cell and tangential electric fields $E_{x}, E_{y}$ defined on the corresponding cells' edges, as shown in Fig. 17. Each cell is characterized by a vector position $(i, j)$. In such staggered mesh, total magnetic flux is identically conserved when we evolve magnetic fields through Faraday's equation.

At $t=0$, we populate our 2D grid with a particular force-free magnetostatic field configuration $B_{z}(i, j ; t=0)$ that can be anything (i.e., zero, dipole-like of any direction and strength, quadrupole-like, etc.). Our aim is to impose a particular horizontal electric field distribution that will asymptotically evolve $B_{z}$ toward a target magnetic field configuration $B_{T}(i, j)$. We chose the following procedure: given the photospheric magnetic field distribution $B_{z}(i, j ; t)$ at each time step $t$, we scan the full photospheric grid $(i, j)$, and at each cell position we add four electric field components at the four edges of the cell such that

$$
\begin{aligned}
E_{x}(i, j ; t) & \rightarrow E_{x}(i, j ; t)-f\left[B_{T}(i, j)-B_{z}(i, j ; t)\right], \\
E_{y}(i, j ; t) & \rightarrow E_{y}(i, j ; t)+f\left[B_{T}(i, j)-B_{z}(i, j ; t)\right], \\
E_{x}(i, j+1 ; t) & \rightarrow E_{x}(i, j+1 ; t)+f\left[B_{T}(i, j)-B_{z}(i, j ; t)\right], \\
E_{y}(i+1, j ; t) & \rightarrow E_{y}(i+1, j ; t)-f\left[B_{T}(i, j)-B_{z}(i, j ; t)\right],
\end{aligned}
$$



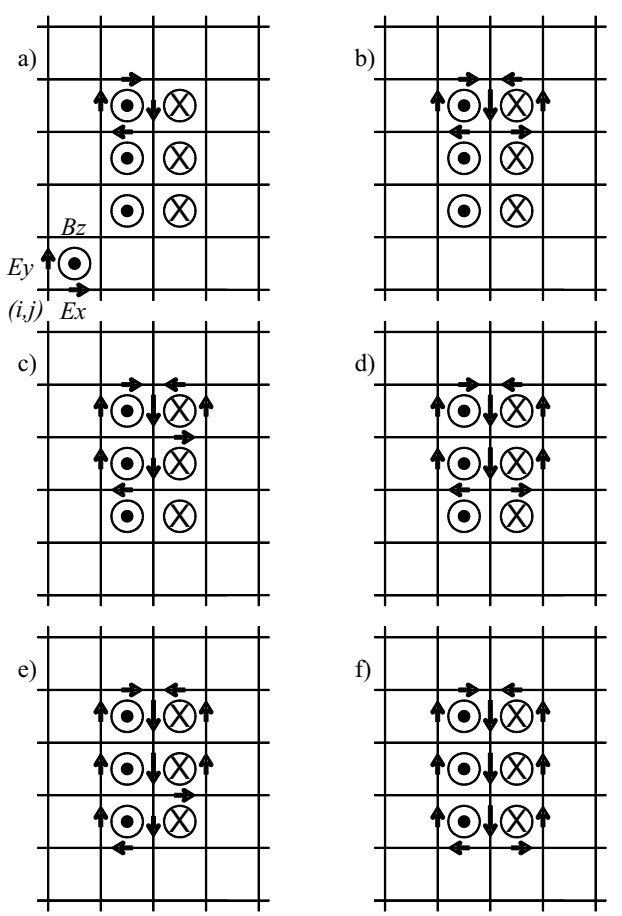

Figure 1. Implementation of electric field update algorithm (eqs. 10) at $t=0$ in a 2D staggered cartesian grid. Electric fields are defined along grid edges. Magnetic fields are defined in cell centers. Nonzero equal-magnitude target magnetic field $B_{T}(i, j)$ only at positions shown with circles. Circles with dots: $B_{T}$ outwards from the plane. Circles with crosses: $B_{T}$ into the plane. The grid is scanned left to right, top to bottom in frames a-f respectively.

as shown in Fig. Th. Here $f$ is a free positive numerical factor used to adjust the rate of convergence. We chose $f=0.5$, as convergence becomes numerically unstable for $f>0.5$ and too slow for $f<0.5$. At each time step $t$, we start with $E_{x}(i, j ; t)=E_{y}(i, j ; t)=0$ everywhere, and then scan the $2 \mathrm{D}$ grid and update electric fields according to eqs. (10). If we only had one computational grid-cell, the $z$ component of Faraday's equation (eq. 2) would be written in a first-order discretized form as follows:

$$
\frac{\partial B_{z}(t)}{\partial t}=\frac{4 c f\left[B_{T}-B_{z}(t)\right]}{\delta} .
$$

This equation would be integrated numerically to obtain the asymptotic solution $B_{z}(t \rightarrow \infty)=B_{T}$. When the algorithm is applied sequentially to the full horizontal grid, though, electric fields on edges that correspond to neighboring cells will be updated twice as the algorithm is applied in both cells. In the sketch of Fig. 1 1b-f, one can see how the electric fields of neighboring cells are updated for a simple magnetic field distribution consisting of three plus three neighboring cells with equal and opposite magnetic fields. An important point to make here is that the process can be generalized in spherical coordinates (a step to take in the future) besides the Cartesian implementation shown here. 
The above 2D procedure can be directly generalized in 3D. We use a cubic cartesian grid in order to avoid numerical artifacts associated with the axis of a spherical or cylindrical coordinate system. Note that in such a grid, the photosphere nowhere coincides with any of the grid cell phases. We define as our solar boundary surface the outer surface of the largest cartesian volume lying entirely within $r_{\odot}$. Given a target photospheric radial magnetic field distribution $B_{T}\left(r_{\odot}, \theta, \phi\right) \hat{\mathbf{r}}$ in heliocentric spherical coordinates (see the discussion in $\S 4$ on how $B_{T}$ is obtained from a Carrington magnetogram), we define the target magnetic field at the center of every boundary cell surface at positions $(x, y, z)$ as the projection perpendicular to that surface of the field $B_{T}\left(r_{\odot}, \theta, \phi\right)\left(r_{\odot} / r\right)^{2} \hat{\mathbf{r}} \equiv$ $\left(B_{T x}, B_{T y}, B_{T z}\right)$ (for practical purposes, it is assumed here that the magnetic field inside a certain depth below the photosphere is purely radial, since our cartesian grid does not trace too accurately the solar surface). As before, we then scan the boundary surface and update electric fields as

$$
\begin{aligned}
E_{x}(i, j, k ; t) & \rightarrow E_{x}(i, j, k ; t)-f\left(B_{T z}(i, j, k)-B_{z}(i, j, k ; t)\right), \\
E_{y}(i, j, k ; t) & \rightarrow E_{y}(i, j, k ; t)+f\left(B_{T z}(i, j, k)-B_{z}(i, j, k ; t)\right), \\
E_{x}(i, j+1, k ; t) & \rightarrow E_{x}(i, j+1, k ; t)+f\left(B_{T z}(i, j, k)-B_{z}(i, j, k ; t)\right), \\
E_{y}(i+1, j, k ; t) & \rightarrow E_{y}(i+1, j, k ; t)-f\left(B_{T z}(i, j, k)-B_{z}(i, j, k ; t)\right), \\
E_{y}(i, j, k ; t) & \rightarrow E_{y}(i, j, k ; t)-f\left(B_{T x}(i, j, k)-B_{x}(i, j, k ; t)\right), \\
E_{z}(i, j, k ; t) & \rightarrow E_{z}(i, j, k ; t)+f\left(B_{T x}(i, j, k)-B_{x}(i, j, k ; t)\right), \\
E_{y}(i, j, k+1 ; t) & \rightarrow E_{y}(i, j, k+1 ; t)+f\left(B_{T x}(i, j, k)-B_{x}(i, j, k ; t)\right), \\
E_{z}(i, j+1, k ; t) & \rightarrow E_{z}(i, j+1, k ; t)-f\left(B_{T x}(i, j, k)-B_{x}(i, j, k ; t)\right), \\
E_{x}(i, j, k ; t) & \rightarrow E_{x}(i, j, k ; t)-f\left(B_{T y}(i, j, k)-B_{y}(i, j, k ; t)\right), \\
E_{z}(i, j, k ; t) & \rightarrow E_{z}(i, j, k ; t)+f\left(B_{T y}(i, j, k)-B_{y}(i, j, k ; t)\right), \\
E_{x}(i, j, k+1 ; t) & \rightarrow E_{x}(i, j, k+1 ; t)+f\left(B_{T y}(i, j, k)-B_{y}(i, j, k ; t)\right), \\
E_{z}(i+1, j, k ; t) & \rightarrow E_{z}(i+1, j, k ; t)-f\left(B_{T y}(i, j, k)-B_{y}(i, j, k ; t)\right),
\end{aligned}
$$

for the corresponding boundary cells that face in the $z, x$, and $y$ direction respectively. As in 2D, each boundary electric field component is updated twice from its two neighboring boundary surface grid cells.

To test our algorithm we have used a synoptic magnetogram mainly referring to Carrington rotation 2009 recorded by the Michelson-Doppler Imager (MDI; Scherrer et al., 1995) onboard the Solar and Heliospheric Observatory (SoHO). The choice was deliberate: Carrington rotation 2009 corresponds to the period of October-November 2003 (the so-called 'Halloween 2003' period) when the Sun exhibited unusually high eruptive activity and the solar active-region belt was populated by several very complex active regions (perhaps an active-region nest, as proposed by Zhou et al., 2007). Using a solar dipole aligned with the dipole component of the given magnetogram as the initial condition, the photospheric magnetic field evolution toward the target is depicted in Fig. 2. Here, the entire 
solar disk is represented in $(\theta, \phi)$ heliocentric coordinates. Photospheric snapshots are labelled by the number $n$ of numerical time-integration steps, with the target magnetogram denoted with an infinite iteration number $(n=\infty)$. One may notice that the field evolution mimics flux emergence/submergence through the solar photosphere. Our procedure quickly reproduces the complex active regions present in the disk, but takes much longer to reproduce the weaker, large-scale field distribution in the polar regions.

At this point, we should discuss the spatial resolution of our computational grid. Ideally we would like our uniform cubic grid to be able to capture both the details of the 'small-scale', strong active-region magnetic field and the largescale structure of the much weaker coronal field. The first requirement calls for a size element $\delta$ comparable to the resolution of the SoHO/MDI synoptic magnetogram, that is, $\delta \sim 0.01 r_{\odot}$ or $0.5^{\circ}$ per pixel. Coupled with the second requirement that dictates a spatial extent of $\sim 1.5 r_{\odot}$ from the photosphere, it would give rise to a computational domain of $\sim 10^{8}$ cubic elements. For our calculation, that is performed on a typical desktop workstation, this grid size is infeasible. We thus reduced the original resolution of the synoptic magnetogram by a factor of $4\left(\delta \sim 0.04 r_{\odot}\right.$ or $\sim 2^{\circ}$ per pixel $)$. If we were extrapolating for only a part of the solar disk, say, an active region, this restriction would be much less stringent, of course. As a result, the target synoptic magnetogram in Fig. 2 is shown with resolution degraded by a factor of 4 . Although we cannot be certain about the degradation factors of other methods, say, the magnetostatic or magnetohydrostatic methods of T. Wiegelmann and colleagues, significant spatial degradation is, indeed, a necessity that one has to deal with when calculating the global solar magnetic field. The numerical time-integration step of our simulation is $0.5 \delta / c=0.05 \mathrm{sec}$. Our simulations need about 24 hours on a desktop Intel i7 quad-core workstation to complete 10,000 time-integration steps.

\section{The coronal magnetic field}

Given the above boundary condition for the photospheric electric field we now follow the respective magnetic field evolution in the corona. This is treated as a pure electrodynamic problem that can be described through the formalism of FFE. We emphasize, though, that this is not a vacuum electrodynamic problem since electric currents and electric charges are allowed to develop everywhere in the corona to comply with the ideal MHD condition, eq. (44).

Electrodynamic waves will traverse the computational region informing the corona that the photosphere is changing. As they do so, they leave behind a modified coronal electric and magnetic field distribution. Similar waves are continuously generated during photospheric magnetic flux emergence/submergence and travel out to larger heights in the corona to be absorbed in the non force-free region of the solar wind. This absorption is implemented numerically through a Perfectly Matched Layer (hereafter PML; Berenger, 1994, 1996, see also Kalapotharakos and Contopoulos, 2009), that is, a non-reflecting, perfectly absorbing boundary that mimics open space. 


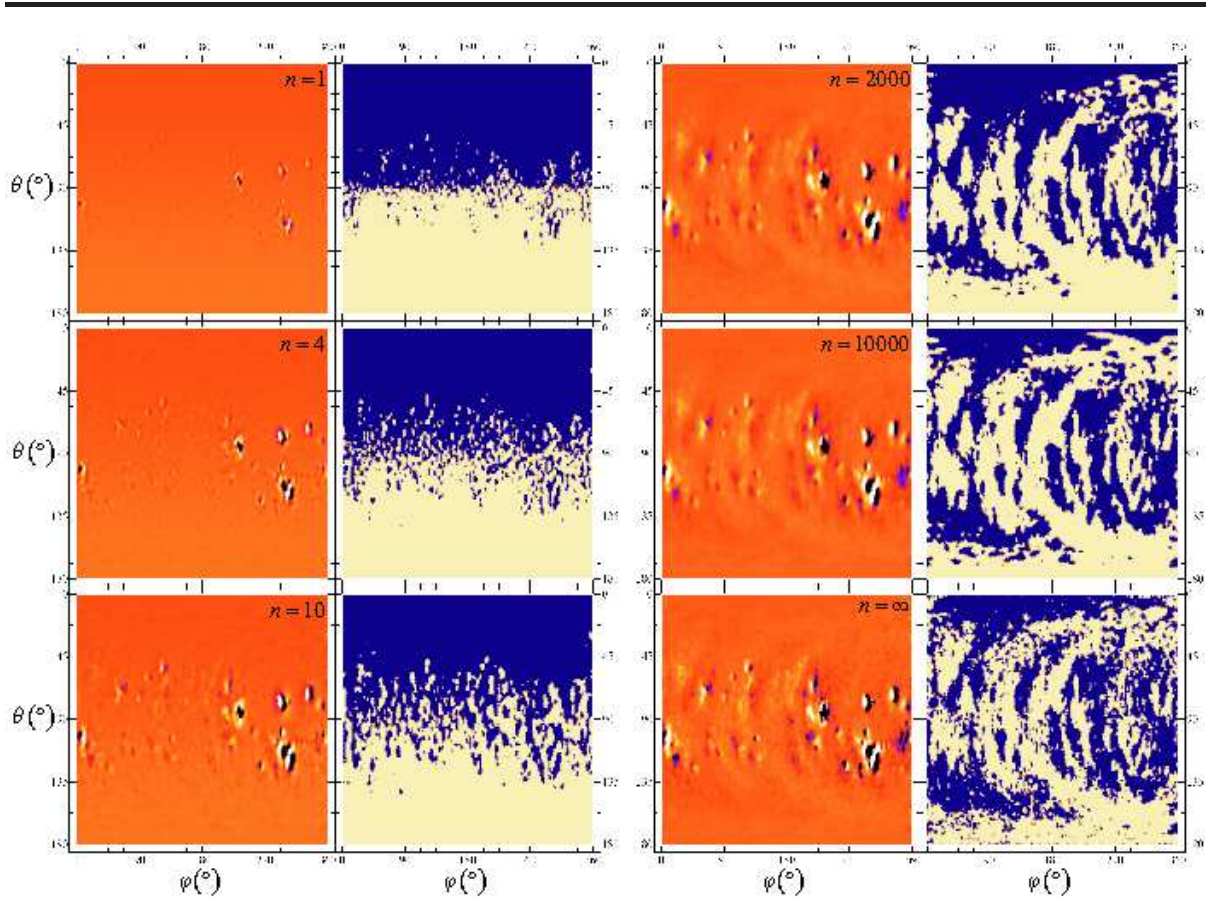

Figure 2. Evolution of the simulated photospheric normal field component in heliocentric $(\theta, \phi)$ coordinates. Sub-plots labelled according to time-integration step. The same photospheric instances are shown with two different color scales. Left: linear color scale from $-1 \mathrm{kG}$ to $+1 \mathrm{kG}$. Right: brighter areas: $B_{r}>0$; darker areas: $B_{r}<0$. Initial configuration: dipolar, with $B_{r}\left(\theta=0^{\circ}\right)=-0.85 \mathrm{G}$. Target magnetogram labelled " $\infty$ ". This time-sequence results in the equilibrium solution shown in Fig. 3

As the distribution of the radial photospheric field approaches that implied by the target magnetogram, the photospheric electric fields will gradually fade. As a consequence, the coronal electrodynamic waves and electric fields will also fade, and the coronal magnetic field will gradually approach a force-free magnetostatic configuration satisfying the ideal MHD condition. In doing so, a nonlinear coronal current network develops. In Figs. 3, 4 we show two examples of global coronal solutions reached for two different initial coronal field configurations. In the lower right panels we plot the evolution with time-integration step of i) the total magnetic energy $\int B^{2} \mathrm{~d} V$ over our computational volume $V$ normalized to its asymptotic value obtained after $n=10,000$ time-integration steps (dashed line), ii) the average sine of the angle between the electric current $\mathbf{J} \propto \nabla \times \mathbf{B}$ and the magnetic field $\mathbf{B}$ weighted by the magnitudes of $\mathbf{J}$ and B (dotted line), and iii) the average absolute difference between the target and actual magnetograms normalized to the average magnitude of the target photospheric magnetic field (solid line). As can be seen, the first two monitors approach numerical convergence to within $10 \%$ in about 500 time-integration steps, but the third monitor takes roughly ten times as many steps to drop below the level of $10 \%$. Indeed, the photospheric magnetic field needs longer integration times to settle to its asymptotic value, implying that surface electric fields take 

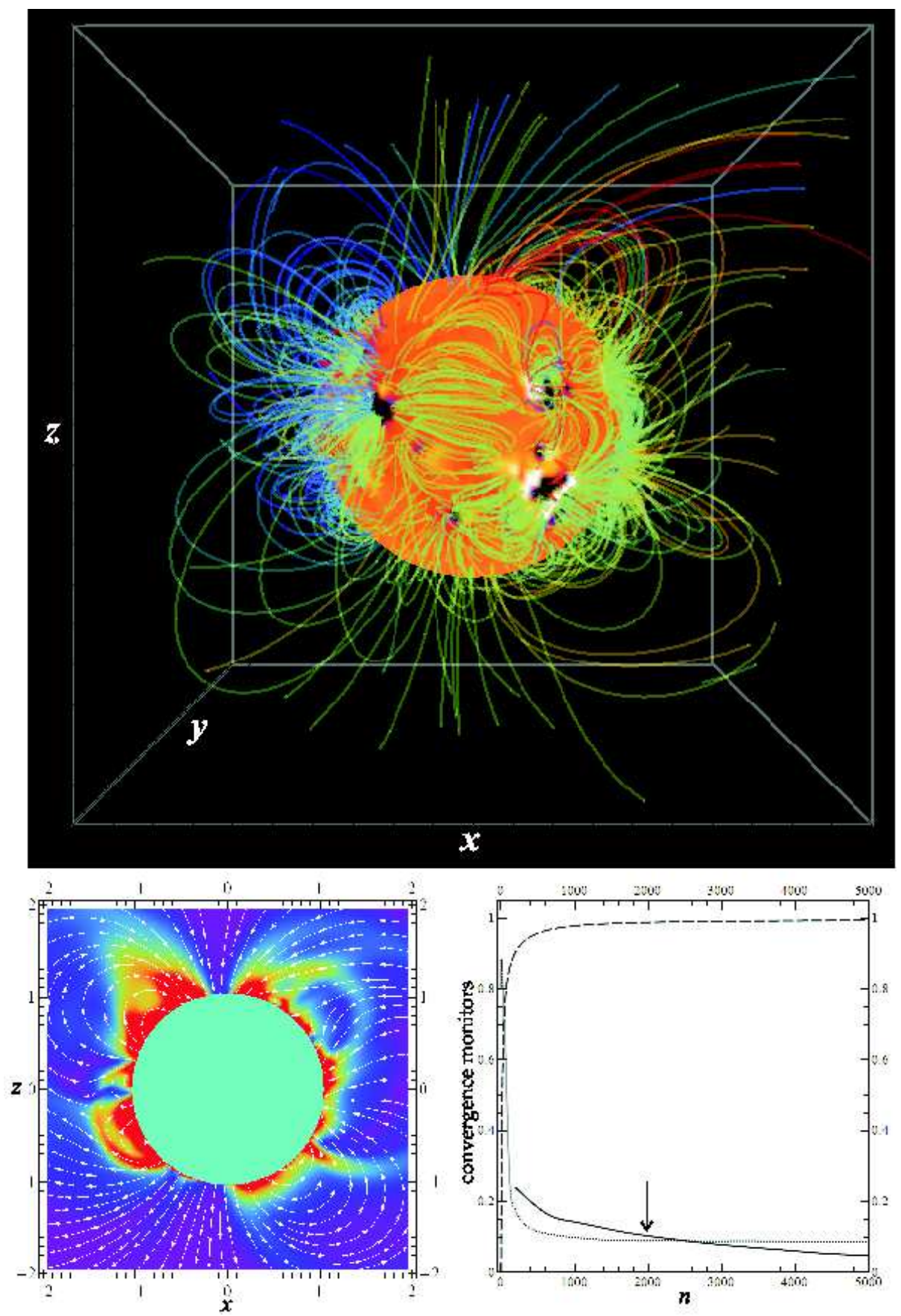

Figure 3. Global nonlinear force-free coronal configuration corresponding to the target photospheric radial field of Carrington rotation 2009. Initial dipole polar magnetic field: $B_{r}\left(\theta=0^{\circ}\right)=-0.85 \mathrm{G}$. Top panel: $3 \mathrm{D}$ configuration of magnetic field lines as viewed from the direction $\phi=0^{\circ}$. Field-line color: twist parameter $\alpha \equiv(\nabla \times \mathbf{B}) / \mathbf{B}$. Photospheric color scale as in Fig. 2 Lower left panel: final configuration of magnetic fields projected onto a 2D meridional cut along $\phi=90^{\circ}$. Color scale: absolute value of the electric current $J$. Lower right panel: evolution of convergence monitors with numerical time-integration step. Dashed line: magnetic field energy normalized to its asymptotic value attained after 10,000 steps. Dotted line: average weighted sine of the angle between $\mathbf{J}$ and $\mathbf{B}$. Solid line: average absolute difference between target and actual magnetograms over average absolute target magnetic field. Arrow: numerical integration step that corresponds to the plotted solution (this is when all convergence monitors fall below $10 \%$ of their asymptotic value). 

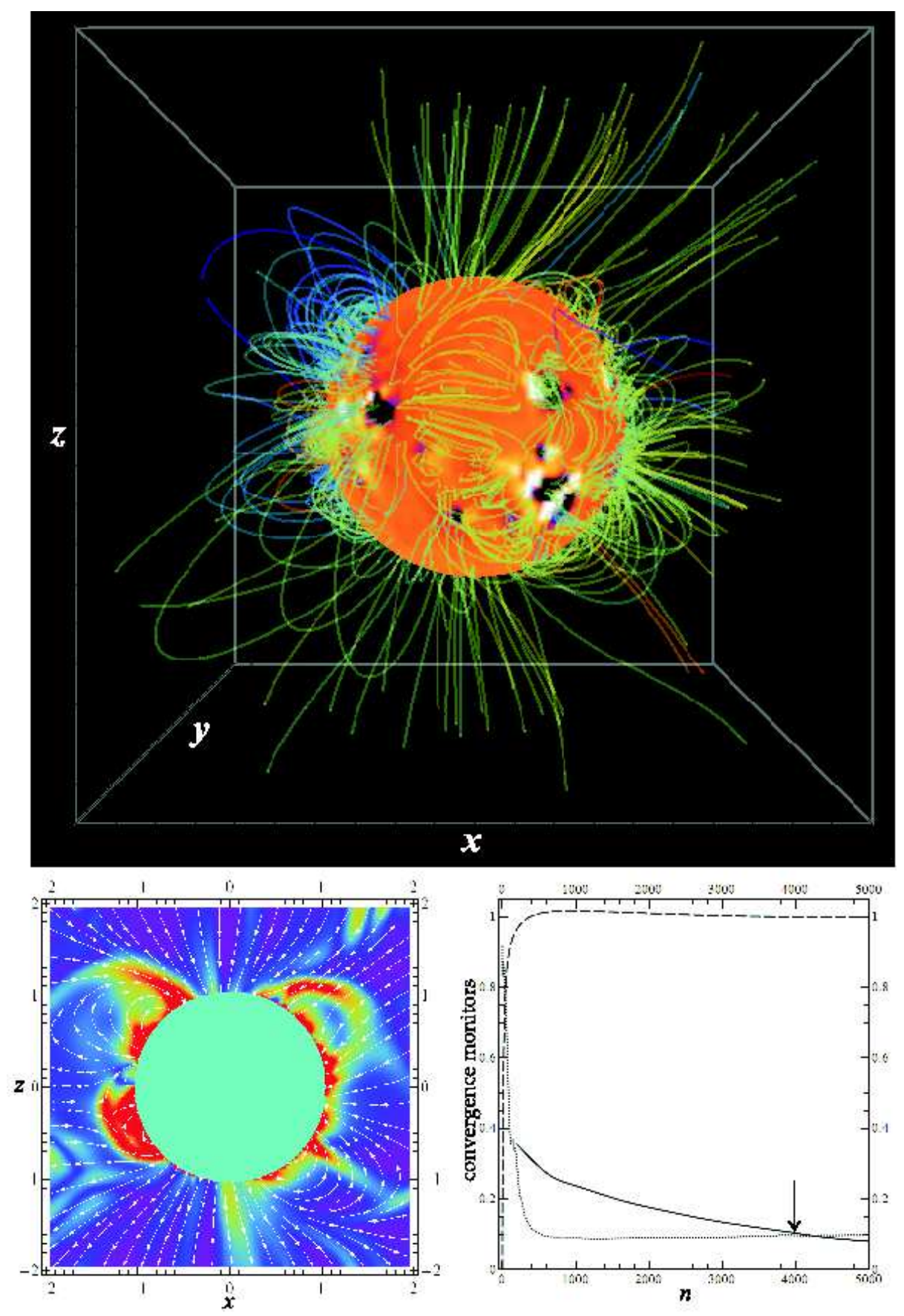

Figure 4. Same as figure 3 but with initial dipole polar magnetic field $B_{r}\left(\theta=0^{\circ}\right)=-2 \mathrm{G}$. $3 \mathrm{D}$ field lines shown have the same footpoints as in Fig. 3 


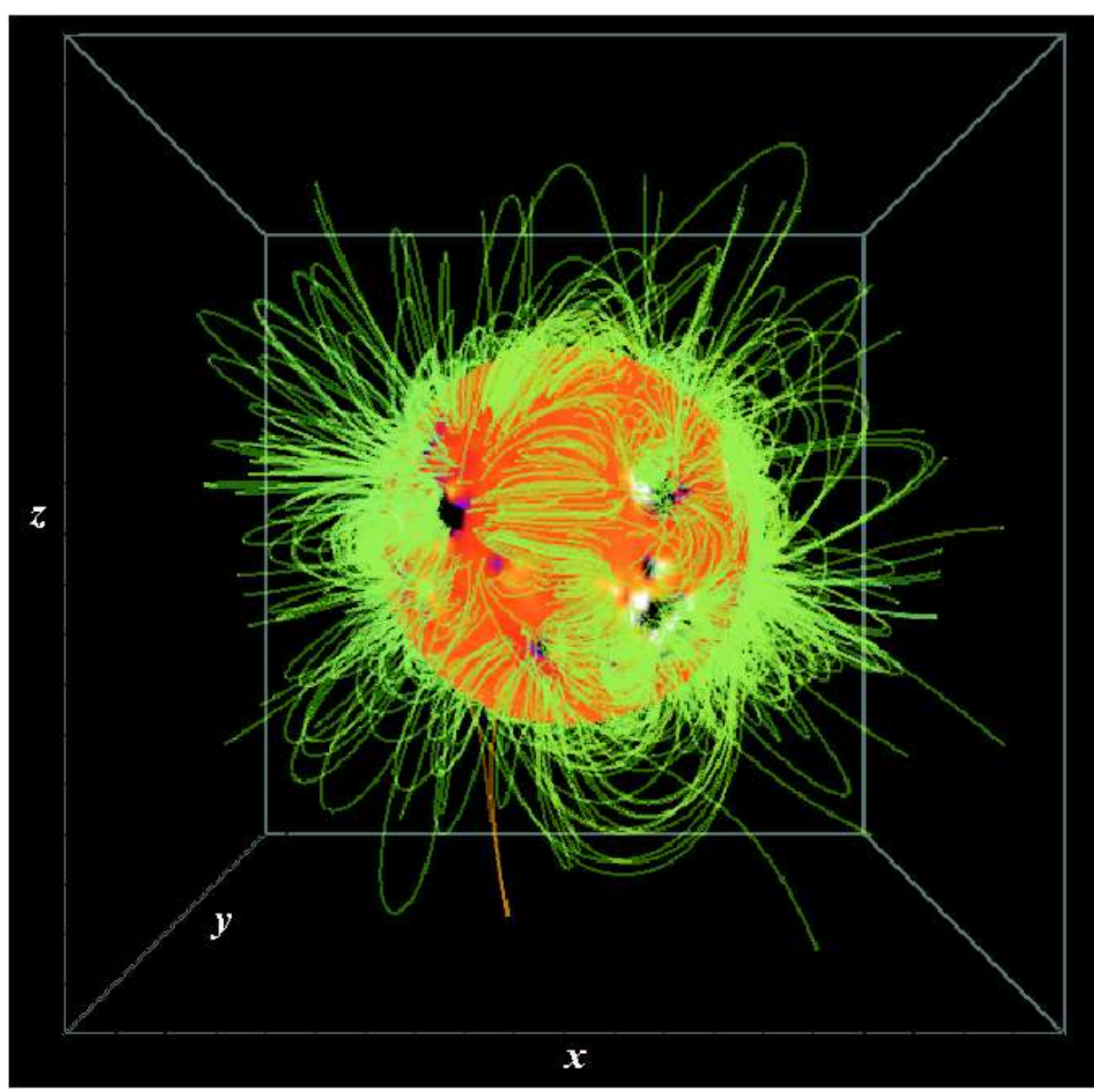

Figure 5. Coronal configuration with nearly zero electric currents (potential) that corresponds to the same target photosphere as in figs. 3 \& 4 obtained after 10,000 time integration steps.

longer times to fade out. Currently we consider as equilibrium solution the state in which all convergence monitors fall within $10 \%$ of their asymptotic value. This practice will be reconsidered in future efforts. In the lower left panels we show the final configuration of magnetic fields projected onto a $2 \mathrm{D}$ meridional cut along $\phi=90^{\circ}$, colored by the magnitude of the field-aligned electric current. In the top panels we show a 3D configuration of magnetic field lines as observed from the direction $\phi=0^{\circ}$, colored according to the twist parameter $\alpha \equiv(\nabla \times \mathbf{B}) / \mathbf{B}$ (magnetic field line color is redish where $\mathbf{J}$ is parallel to $\mathbf{B}$, blueish where $\mathbf{J}$ is anti-parallel to $\mathbf{B}$, and light green where $\mathbf{J} \approx 0$ ).

In Fig. 3, we begin with a dipole magnetic field counter-aligned with the solar axis with $B_{r}\left(\theta=0^{\circ}\right)=-0.85 \mathrm{G}$, and aim for an actual global photospheric magnetic field obtained through part of Carrington magnetogram 2009. The chosen initial polar magnetic field value corresponds to the average one in the given magnetogram. By choosing this particular value we 'assist' the longerterm numerical convergence of our code. As can be seen in Fig. 3, the target 
magnetogram is reproduced to within $10 \%$ in about 2,000 time integration steps (see also time sequence of Fig. 22). This is when we plot the global coronal solution shown in Fig. 3. The mean weighted sine of the angle between the electric current and the magnetic field vectors is $\leq 0.1$, and the values of $|\nabla \cdot \mathbf{B}| /\left[\left(\frac{\partial B_{x}}{\partial x}\right)^{2}+\left(\frac{\partial B_{y}}{\partial y}\right)^{2}+\left(\frac{\partial B_{z}}{\partial z}\right)^{2}\right]^{\frac{1}{2}}$ are on the order of $10^{-5}$. The $\alpha$ parameter values are in the range $\sim \pm 10^{-3} \mathrm{Mm}^{-1}$ (3 standard deviations away from its zero mean value). Notice that such twist is not unreasonable in the solar corona. Here we should clarify that, although the photosphere has relaxed to its equilibrium configuration, continuing numerical integration beyond this stage results in a gradual disappearance of coronal electric currents due to the numerical diffusion inherent in any numerical code such as this one. In other words, coronal currents are continuously 'eroded' by numerical diffusivity. We know that, by the time we manage to reproduce our target photospheric boundary condition to within $10 \%$, some amount of the coronal electric current will be lost due to numerical diffusivity. Beyond that point, the corona evolves through a sequence of magnetostatic equilibria, each with smaller amount of electric current. After about 10,000 time integration steps, we reach a configuration (Fig. 5) with very small coronal electric currents $\left(\alpha \sim \pm 10^{-4} \mathrm{Mm}^{-1}\right)$. This current-free configuration is uniquely determined for a closed volume or for a lower boundary condition and the semi-infinite space above it (e.g., Aly, 1987), which is our approximation.

Figure 4 is similar to Fig. 3 only the initial magnetic field is dipolar counteraligned with the solar axis with $B_{r}\left(\theta=0^{\circ}\right)=-2 \mathrm{G}$. As before, we reproduce very quickly the main photospheric active regions, but now the polar photospheric regions take longer to form, within about 4,000 time integration steps. One might expect that, due to the slower convergence, the resulting coronal electric currents are now slightly weaker than before ( $\alpha$ values of the order $\pm 0.75 \times$ $10^{-3} \mathrm{Mm}^{-1}$ ), and the two coronal configurations are somewhat different. If we continue the time integration for much longer times, coronal electric currents will also fade gradually, and the coronal configuration will approach the same potential configuration shown in Fig. 圀,

We also tested our algorithm in a much simpler configuration, namely that of a solar dipole without magnetic flux in the active-region belt. We used various initial configurations (zero or dipolar). In all cases, the equilibrium solution is almost indistinguishable from the vacuum magnetostatic dipole (potential field), with very small field-aligned electric currents ( $\alpha$ values of the order $\pm 10^{-4} \mathrm{Mm}^{-1}$ ). It seems that the absence of magnetic flux in the active-region belt gives rise to weak coronal electric currents and by the time the photosphere relaxes to its given boundary configuration numerical diffusivity has enough time to dissipate them effectively. In all cases, the average weighted value of the sine of the angle between the electric current and the magnetic field is $<0.1$, and the maximum value of $|\nabla \cdot \mathbf{B}| /\left[\left(\frac{\partial B_{x}}{\partial x}\right)^{2}+\left(\frac{\partial B_{y}}{\partial y}\right)^{2}+\left(\frac{\partial B_{z}}{\partial z}\right)^{2}\right]^{\frac{1}{2}}$ is of the order $10^{-5}$. We obtained similar results when we considered different dipole orientations with respect to the axes of our numerical grid. This is evidence that our results are grid independent. 


\section{Discussion and conclusions}

Both solutions shown in Figs. 3 and 4 represent force-free magnetostatic configurations satisfying the ideal-MHD condition that correspond to the same magnetogram on the solar photosphere, therefore, they both represent solutions to our problem. The potential solution shown in Fig. [5] also represents a solution to our problem. Nature, however, chooses only one particular configuration. Our numerical approach suggests that our reconstructed field retains some memory of the prehistory of its evolution: it depends on the initial global configuration and on the course toward equilibrium. This is analogous to what we suspect happens in the real Sun as well, when different solar cycles succeed one another. If the target boundary does not have any well-formed active regions, i.e. like in a solar-minimum configuration, our simulation results in the unique potential-field solution between the photosphere and the source surface or for the semi-infinite space above the photosphere. This is also what full-fledged magnetohydrodynamic global models give, in this case approaching the results of potential-field models (Riley et al., 2006). Obviously there is no straightforward answer to the question of how to choose one particular coronal field configuration from another. One way could be to introduce additional control monitors such as magnetic free energy in our computational volume or the Poynting flux through the photospheric boundary. Another way would be to make use of full vector magnetograms whenever those are available (for example, in eqs. (12) we could involve the three magnetic field components on the boundary toward the target components $\left(B_{T x}, B_{T y}, B_{T z}\right)$ of the observed field vector $\mathbf{B}$; not only its radial component $\left.B_{r} \hat{\mathbf{r}}\right)$. This major issue, that could fully constrain our solution, will be addressed in a forthcoming publication.

Finally, we address a problem associated with the observational method via which the radial component of the photospheric magnetic field $B_{T}\left(r_{\odot}, \theta, \phi\right)$ is inferred from measurements of the line-of-sight magnetic field $B_{L O S}\left(r_{\odot}, \theta, \phi\right)$. We will only consider here the method applied to a Carrington synoptic magnetogram, although our discussion below also applies to local observations of particular photospheric active regions, as well. In a Carrington magnetogram we are observing the photosphere along solar meridional sections that contain our line of sight. As the Sun rotates, the full photosphere is covered and a global magnetogram is obtained under the assumption that the magnetic field structure remains unchanged during a full solar revolution (obviously, this is not quite true, but is the best one can do with present day satellite technology, avoiding also further interference with observational data). In order to obtain the radial magnetic field, a further assumption is made, namely that the magnetic field at the base of the corona is everywhere perpendicular to the photosphere, i.e.,

$$
B_{T}\left(r_{\odot}, \theta, \phi\right)=\frac{B_{L O S}\left(r_{\odot}, \theta, \phi\right)}{\sin \theta} .
$$

This second assumption is also not totally accurate, but is the best one can do without knowledge of the photospheric field vector. The orientation of this field can be inferred from a full 3D reconstruction of the global coronal magnetic field, 
and this is precisely what we are doing in the present work. We, therefore, propose to include in a future publication one more step in the iteration procedure implemented along the photosphere, namely,

i) At time $t=0$, start with a purely radial target for the normal photospheric field as given by eq. (13).

ii) Apply the numerical integration steps and calculate the interim line-of-sight component $B_{\operatorname{LOS}(\text { num })}\left(r_{\odot}, \theta, \phi ; t\right)$. In general, this will be different from

$B_{r}\left(r_{\odot}, \theta, \phi ; t\right) \sin \theta$ because a nonzero horizontal magnetic field now develops in the photosphere.

iii) Rescale the target field $B_{T}\left(r_{\odot}, \theta, \phi\right)$ by a factor reflecting the development of horizontal fields on the boundary, i.e.,

$$
\begin{aligned}
B_{T}\left(r_{\odot}, \theta, \phi\right) & \rightarrow \frac{B_{L O S}\left(r_{\odot}, \theta, \phi\right)}{\sin \theta} \cdot\left(\frac{B_{r}\left(r_{\odot}, \theta, \phi ; t\right) \sin \theta}{B_{L O S(\text { num })}\left(r_{\odot}, \theta, \phi ; t\right)}\right) \\
& =B_{L O S}\left(r_{\odot}, \theta, \phi\right) \cdot\left(\frac{B_{r}\left(r_{\odot}, \theta, \phi ; t\right)}{B_{L O S(\text { num })}\left(r_{\odot}, \theta, \phi ; t\right)}\right) .
\end{aligned}
$$

Here, the geometric term $\sin \theta$, reflecting the assumption of purely radial field on the boundary in eq. (13), is replaced by the more general term $B_{L O S(\text { num })} / B_{r}$.

The above iterative procedure, implemented together with eqs. (12), continuously redefines the target radial photospheric field, $B_{T}$, gradually relaxing the assumption of purely radial fields in the photosphere. To our knowledge, this is the first time that the full 3D coronal field geometry has been taken into account in de-projecting the photospheric magnetic field.

The quest toward choosing the most realistic global reconstruction of the solar coronal magnetic field at the lowest possible computational expense is, obviously, still ongoing. Despite the non-uniqueness of our solution we believe that the proposed method is interesting and may even turn out to become promising, due to the following two reasons: firstly, ours is the first technique that reaches a nonlinear force-free solution using only the radial photospheric field that is readily available from various sources. Attempting to match our force-free tangential evolution in the photosphere with the tangential component provided by SOLIS/VSM or will be provided by SDO/HMI full-disk vector magnetograms may further constrain, or determine, hopefully, our solution. Secondly, our method alleviates the need for preprocessing the forced photospheric fields to better comply with a coronal force-free solution. Preprocessing introduces additional assumptions (e.g., Tadesse, Wiegelmann, and Inhester, 2009) so the fact that we asymptotically reach the target synoptic magnetogram at the same time ensuring a force-free solution above each interim boundary configuration may prove advantageous. Concerning our non-uniqueness problem, we will consider, classify, examine, and implement various revisions that may potentially present an opportunity to improve our global solar magnetic field reconstruction method.

Acknowledgements This work uses synoptic solar magnetograms from SoHO/MDI. SoHO is a project of international cooperation between ESA and NASA. 


\section{References}

Altschuler, M.D., Newkirk, G.: 1969, Magnetic Fields and the Structure of the Solar Corona. I: Methods of Calculating Coronal Fields. Solar Phys. 9, 131 - 149. doi 10.1007/BF00145734

Aly, J.J.: 1987, On the uniqueness of the determination of the coronal potential magnetic field from line-of-sight boundary conditions. Solar Phys. 111, 287-296. doi $10.1007 /$ BF00148521

Berenger, J.: 1996, Three-Dimensional Perfectly Matched Layer for the Absorption of Electromagnetic Waves. Journal of Computational Physics 127, 363-379. doi $10.1006 /$ jcph.1996.0181

Berenger, J.: 1994, A Perfectly Matched Layer for the Absorption of Electromagnetic Waves. Journal of Computational Physics 114, 185 - 200. doi 10.1006/jcph.1994.1159.

Georgoulis, M.K., LaBonte, B.J.: 2004, Vertical Lorentz Force and Cross-Field Currents in the Photospheric Magnetic Fields of Solar Active Regions. Astrophys. J. 615, 1029-1041. doi $10.1086 / 424501$

Gruzinov, A.: 1999, Stability in Force-Free Electrodynamics. ArXiv Astrophysics e-prints.

Henney, C.J., Keller, C.U., Harvey, J.W., Georgoulis, M.K., Hadder, N.L., Norton, A.A., Raouafi, N., Toussaint, R.M.: 2009, SOLIS Vector Spectromagnetograph: Status and Science. In: S. V. Berdyugina, K. N. Nagendra, \& R. Ramelli (ed.) Astronomical Society of the Pacific Conference Series, Astronomical Society of the Pacific Conference Series 405, 47.

Kalapotharakos, C., Contopoulos, I.: 2009, Three-dimensional numerical simulations of the pulsar magnetosphere: preliminary results. Astron. Astrophys. 496, 495-502. doi $10.1051 / 0004-6361: 200810281$

Linker, J.A., Mikić, Z., Biesecker, D.A., Forsyth, R.J., Gibson, S.E., Lazarus, A.J., Lecinski, A., Riley, P., Szabo, A., Thompson, B.J.: 1999, Magnetohydrodynamic modeling of the solar corona during Whole Sun Month. J. Geophys. Res. 104, 9809-9830. doi $10.1029 / 1998 J A 900159$

Luhmann, J.G., Li, Y., Arge, C.N., Gazis, P.R., Ulrich, R.: 2002, Solar cycle changes in coronal holes and space weather cycles. Journal of Geophysical Research (Space Physics) 107, 1154. doi:10.1029/2001JA007550

Metcalf, T.R., Jiao, L., McClymont, A.N., Canfield, R.C., Uitenbroek, H.: 1995, Is the solar chromospheric magnetic field force-free? Astrophys. J. 439, 474-481. doi 10.1086/175188

Riley, P., Linker, J.A., Mikić, Z.: 2001, An empirically-driven global MHD model of the solar corona and inner heliosphere. J. Geophys. Res. 106, 15889-15902. doi $10.1029 / 2000$ JA000121

Riley, P., Linker, J.A., Mikić, Z., Lionello, R., Ledvina, S.A., Luhmann, J.G.: 2006, A Comparison between Global Solar Magnetohydrodynamic and Potential Field Source Surface Model Results. Astrophys. J. 653, 1510-1516. doi 10.1086/508565

Roumeliotis, G.: 1996, The "Stress-and-Relax" Method for Reconstructing the Coronal Magnetic Field from Vector Magnetograph Data. Astrophys. J. 473, 1095. doi 10.1086/178219

Roussev, I.I., Gombosi, T.I., Sokolov, I.V., Velli, M., Manchester, W. IV, DeZeeuw, D.L., Liewer, P., Tóth, G., Luhmann, J.: 2003, A Three-dimensional Model of the Solar Wind Incorporating Solar Magnetogram Observations. Astrophys. J. 595, L57-L61. doi $10.1086 / 378878$

Ruan, P., Wiegelmann, T., Inhester, B., Neukirch, T., Solanki, S.K., Feng, L.: 2008, A first step in reconstructing the solar corona self-consistently with a magnetohydrostatic model during solar activity minimum. Astron. Astrophys. 481, 827-834. doi $10.1051 / 0004-6361: 20078834$

Schatten, K.H., Wilcox, J.M., Ness, N.F.: 1969, A model of interplanetary and coronal magnetic fields. Solar Phys. 6, $442-455$. doi $10.1007 /$ BF00146478.

Scherrer, P.H., Bogart, R.S., Bush, R.I., Hoeksema, J.T., Kosovichev, A.G., Schou, J., Rosenberg, W., Springer, L., Tarbell, T.D., Title, A., Wolfson, C.J., Zayer, I., MDI Engineering Team: 1995, The Solar Oscillations Investigation - Michelson Doppler Imager. Solar Phys. 162, 129 - 188. doi:10.1007/BF00733429

Schrijver, C.J., De Rosa, M.L.: 2003, Photospheric and heliospheric magnetic fields. Solar Phys. 212, 165 - 200. doi 10.1023/A:1022908504100

Spitkovsky, A.: 2006, Time-dependent Force-free Pulsar Magnetospheres: Axisymmetric and Oblique Rotators. Astrophys. J. 648, L51-L54. doi $10.1086 / 507518$ 
Tadesse, T., Wiegelmann, T., Inhester, B.: 2009, Nonlinear force-free coronal magnetic field modelling and preprocessing of vector magnetograms in spherical geometry. Astron. Astrophys. 508, 421 - 432. doi 10.1051/0004-6361/200912529

Valori, G., Kliem, B., Keppens, R.: 2005, Extrapolation of a nonlinear force-free field containing a highly twisted magnetic loop. Astron. Astrophys. 433, 335-347. doi 10.1051/0004-6361:20042008

Wang, Y., Sheeley, N.R. Jr.: 1992, On potential field models of the solar corona. Astrophys. J. 392, $310-319$. doi $10.1086 / 171430$

Wiegelmann, T.: 2004, Optimization code with weighting function for the reconstruction of coronal magnetic fields. Solar Phys. 219, 87-108. doi 10.1023/B:SOLA.0000021799.39465.36

Wiegelmann, T.: 2007, Computing Nonlinear Force-Free Coronal Magnetic Fields in Spherical Geometry. Solar Phys. 240, 227 - 239. doi 10.1007/s11207-006-0266-3.

Wiegelmann, T., Neukirch, T., Ruan, P., Inhester, B.: 2007, Optimization approach for the computation of magnetohydrostatic coronal equilibria in spherical geometry. Astron. Astrophys. 475, 701 - 706. doi:10.1051/0004-6361:20078244.

Yee, K.: 1966, Numerical solution of inital boundary value problems involving maxwell's equations in isotropic media. IEEE Transactions on Antennas and Propagation 14, $302-307$. doi 10.1109 /TAP.1966.1138693

Zhou, G., Wang, J., Wang, Y., Zhang, Y.: 2007, Quasi-Simultaneous Flux Emergence in the Events of October November 2003. Solar Phys. 244, 13-24. doi $10.1007 /$ s11207-007-9032-4. 
SOLA: Lowresolution.tex; 27 September 2018; 12:23; p. 18 